PROCEEDINGS OF THE

AMERICAN MATHEMATICAL SOCIETY

Volume 136, Number 8, August 2008, Pages 2997-3006

S 0002-9939(08)09399-4

Article electronically published on April 7, 2008

\title{
A SHARP UPPER BOUND FOR THE FIRST DIRICHLET EIGENVALUE AND THE GROWTH OF THE ISOPERIMETRIC CONSTANT OF CONVEX DOMAINS
}

\author{
PEDRO FREITAS AND DAVID KREJČIŘÍK \\ (Communicated by Carmen C. Chicone)
}

\begin{abstract}
We show that as the ratio between the first Dirichlet eigenvalues of a convex domain and of the ball with the same volume becomes large, the same must happen to the corresponding ratio of isoperimetric constants. The proof is based on the generalization to arbitrary dimensions of Pólya and Szegö's 1951 upper bound for the first eigenvalue of the Dirichlet Laplacian on planar star-shaped domains which depends on the support function of the domain.

As a by-product, we also obtain a sharp upper bound for the spectral gap of convex domains.
\end{abstract}

\section{INTRODUCTION}

Let $\Omega$ be a bounded domain (i.e. an open connected set) in the $d$-dimensional Euclidean space $\mathbb{R}^{d}$, with $d \geq 1$, and denote by $\lambda_{1}(\Omega)$ the first eigenvalue of the Dirichlet Laplacian in $L^{2}(\Omega)$. The Faber-Krahn inequality provides a lower bound for $\lambda_{1}(\Omega)$, namely,

$$
\lambda_{1}(\Omega) \geq \lambda_{1}\left(B_{1}\right)\left(\frac{\left|B_{1}\right|}{|\Omega|}\right)^{2 / d},
$$

where $B_{1}$ is the $d$-dimensional ball of unit radius and the absolute-value signs denote $d$-dimensional Lebesgue measure (later on we use the same notation for $(d-1)$ dimensional Hausdorff measure of the boundary of $\Omega$ as well). Modulus a set of zero capacity, equality in (1) is attained if and only if $\Omega$ is a ball, and it is thus of interest to understand how strong this connection is. More precisely, and assuming for the time being that we have a way of measuring how far a set is from the ball of the same volume in terms of elementary geometric quantities, we would like to answer questions such as the following: if a set is far away from the ball, must its first Dirichlet eigenvalue be much larger than that of the ball of the same volume?

This particular question was given a positive answer in $[\mathrm{G}]$, where the measure of deviation of a convex domain from the ball which was used was based on the support

Received by the editors March 20, 2007, and, in revised form, January 24, 2008.

2000 Mathematics Subject Classification. Primary 58J50, 35P15.

This work was partially supported by FCT, Portugal, through programs POCTI/MAT/60863/2004, POCTI/POCI2010 and SFRH/BPD/11457/2002. The second author was also supported by the Czech Academy of Sciences and its Grant Agency within the projects IRP AV0Z10480505 and A100480501, and by the project LC06002 of the Ministry of Education, Youth and Sports of the Czech Republic.

(C)2008 American Mathematical Society Reverts to public domain 28 years from publication 
function of the domain. More recently, in $[\mathrm{M}, \mathrm{BC}, \mathrm{FMP}$, the authors addressed the question of whether a domain for which $\lambda_{1}(\Omega)|\Omega|^{2 / d}$ is close to the corresponding quantity for the ball must be close to the ball in the sense of Fraenkel asymmetry (i.e. Hausdorff distance if $\Omega$ is convex), again providing a positive answer.

In this paper, we consider the issue of whether having a large first Dirichlet eigenvalue implies being away from the corresponding ball. Using the trivial upper bound $\lambda_{1}(\Omega) \leq \lambda_{1}\left(B_{\rho_{\Omega}}\right)$, where $\rho_{\Omega}$ is the inradius of $\Omega$, it is possible to give an immediate answer to this question in terms of $\rho_{\Omega}$. However, this bound is not very good in general, say for long parallelipipeds, and so our purpose was to obtain a different characterization which would behave better precisely when away from the ball.

The main result of this paper in this direction is the following estimate using the isoperimetric constant as a measure of deviation of $\Omega$ from $B$ :

Theorem 1. Let $\Omega$ be a bounded convex domain of $\mathbb{R}^{d}$. Then

$$
\frac{|\partial \Omega|}{|\Omega|^{1-1 / d}} \geq \frac{|\partial B|}{|B|^{1-1 / d}} \sqrt{\frac{\lambda_{1}(\Omega)}{\lambda_{1}(B)}} \frac{\pi}{2 \sqrt{\lambda_{1}\left(B_{1}\right)}},
$$

where $B$ is the ball of volume $|\Omega|$.

The proof is based on the following result:

Theorem 2. Let $\Omega$ be a bounded convex domain of $\mathbb{R}^{d}$. Then

$$
\lambda_{1}(\Omega) \leq \lambda_{1}\left(B_{1}\right) \frac{|\partial \Omega|}{d \rho_{\Omega}|\Omega|}
$$

This upper bound is a consequence of a stronger upper bound for $\lambda_{1}(\Omega)$ holding in the more general case of star-shaped domains and which we believe to be of interest in its own right ( $c f$. Theorem 3 below). This is an extension to arbitrary dimensions of an upper bound for $\lambda_{1}(\Omega)$ appearing in Pólya and Szegö's 1951 book [PS] in the planar case. As in the case of [G], this bound also depends on the support function of the domain in a non-elementary way. Due to this, we postpone the statement of this result to the next section where we provide the necessary background, its proof then being given in Section 3 .

The proof of Theorem 1, together with a brief discussion of optimality, and other applications of our bounds are given in Section 4 , where, in particular, we obtain a sharp upper bound for the spectral gap. Finally, in the last section we recall some two-dimensional upper bounds and conjectures which will be used for comparison and consider some examples.

\section{AN UPPER BOUND FOR THE FIRST EIGENVALUE OF STAR-SHAPED DOMAINS}

To state Pólya and Szegö's result and its generalization to arbitrary dimension we need to introduce a geometric quantity which measures how far away we are from the ball, and which may be expressed in terms of the support function of the given domain. To be more precise, let $\Omega$ be a star-shaped domain with respect to a point $\xi \in \Omega$, i.e., for each point $x \in \partial \Omega$ the segment joining $\xi$ with $x$ lies in $\Omega \cup\{x\}$ and is transversal to $\partial \Omega$ at the point $x$. Assume now that the boundary $\partial \Omega$ is locally Lipschitz. Then the outward unit normal vector field $N: \partial \Omega \rightarrow \mathbb{R}^{d}$ can 
be uniquely defined almost everywhere on $\partial \Omega$. At those points $x \in \partial \Omega$ for which $N(x)$ is uniquely defined, we introduce the support function

$$
h_{\xi}(x):=(x-\xi) \cdot N(x),
$$

where the dot denotes the standard scalar product in $\mathbb{R}^{d}$. We say that $\Omega$ is strictly star-shaped with respect to the point $\xi \in \Omega$ if $\Omega$ is star-shaped with respect to $\xi$ and the support function is uniformly positive, i.e.,

$$
\underset{x \in \partial \Omega}{\operatorname{essinf}} h_{\xi}(x)>0 \text {. }
$$

In this case, we shall denote by $\omega$ the set of points with respect to which $\Omega$ is strictly star-shaped, and define the following intrinsic quantity of the domain:

$$
F(\Omega):=\inf _{\xi \in \omega} \int_{\partial \Omega} h_{\xi}^{-1} .
$$

Our main result reads as follows:

Theorem 3. Let $\Omega$ be a bounded strictly star-shaped domain in $\mathbb{R}^{d}$ with locally Lipschitz boundary $\partial \Omega$. Then

$$
\lambda_{1}(\Omega) \leq \lambda_{1}\left(B_{1}\right) \frac{F(\Omega)}{d|\Omega|} .
$$

Remark 1. If $d=2$, Theorem 3 coincides with the Pólya-Szegö bound [PS, Sec. 5.6].

Remark 2. Combining the upper bound of Theorem 3 with the Faber-Krahn inequality given in (1), we see that $F(\Omega)$ is bounded from below by

$$
F(\Omega) \geq \frac{d\left|B_{1}\right|^{2 / d}}{|\Omega|^{2 / d-1}},
$$

with equality only when $\Omega$ is a ball. In the two-dimensional case this was shown in $\mathrm{A}$ ] by a different method.

If we now restrict ourselves to convex domains, it is possible to simplify the discussion somewhat. To begin with, we have that the boundary of a convex open subset of $\mathbb{R}^{d}$ is locally Lipschitz ( $c f$. [EE, Sec. V.4.1]). Furthermore, $\omega=\Omega$. Indeed, for any $\xi \in \Omega$ one has

$$
\underset{x \in \partial \Omega}{\operatorname{essinf}} h_{\xi}(x) \geq \operatorname{dist}(\xi, \partial \Omega) \quad \text { if } \Omega \text { is convex, }
$$

which follows from the geometrical meaning of $h_{\xi}(x)$ being the distance from $\xi$ to the tangent space $T_{x}(\partial \Omega)$. Finally, (3) can be used to obtain a simple upper bound to $F(\Omega)$,

$$
F(\Omega) \leq \frac{|\partial \Omega|}{\rho_{\Omega}} \quad \text { if } \Omega \text { is convex, }
$$

where $\rho_{\Omega}$ is the inradius of $\Omega$. This approximation readily establishes Theorem 2 , a weaker version of Theorem 3, but, on the other hand, it allows us to write the upper bound explicitly in terms of more elementary geometric quantities.

Remark 3. Since [BZ, Thm. 35.1.2]

$$
\rho_{\Omega}|\partial \Omega| \leq d|\Omega|,
$$

we see that Theorem 2 is, in general, an improvement to the trivial upper bound given by $\lambda_{1}(\Omega) \leq \lambda_{1}\left(B_{\rho_{\Omega}}\right)$. We note, however, that there are certain classes of 
domains such as triangles and regular polygons for which equality holds in (5) (for a recent discussion of these classes in the two- and three-dimensional cases we refer to [AM1, AM2, respectively). Moreover, for triangles it can be checked directly that we also have equality in (4), so that Theorem 3 is equivalent to Theorem 2 and our method gives just the trivial result in this case (see $\mathrm{A}$ for explicit formulae).

Remark 4 . When the domain is star-shaped but not necessarily convex, the bound given by Theorem 3 may be worse than the trivial bound, as exemplified by the Swiss Cross example considered in [HS]; see Example 4 below.

Although our proof of Theorem 2 holds only for the convex case, we conjecture that this bound holds for general bounded domains in $\mathbb{R}^{d}$.

\section{Proof of Theorem 3}

Henceforth let us assume that $d \geq 2$ (for one-dimensional intervals we clearly have equalities in the upper bounds of Theorems 2 and 3 ).

We need to introduce some notation concerning the geometry of the hypersurface $\partial \Omega$. By assumption, each point $x$ on the boundary $\partial \Omega$ has a neighbourhood (an open subset of $\mathbb{R}^{d}$ ) whose intersection with the boundary, denoted by $V \subset \partial \Omega$, is $C^{0,1}$-diffeomorphic to an open subset $U \subset \mathbb{R}^{d-1}$ by means of a chart $\Gamma: U \rightarrow V$. Having in mind that, by Rademacher's theorem, $\Gamma$ is differentiable almost everywhere in $U$, let $g_{i j}$ denote the coefficients of the metric tensor of $\partial \Omega$ induced by these local diffeomorphisms, i.e.,

$$
g_{i j}:=\left(\partial_{i} \Gamma\right) \cdot\left(\partial_{j} \Gamma\right), \quad i, j \in\{1, \ldots, d-1\} .
$$

Recall that the cross-product $\left[\partial_{1} \Gamma, \ldots, \partial_{d-1} \Gamma\right]$ is perpendicular to $\partial \Omega$ and that its magnitude is equal to the square root of $|g|:=\operatorname{det}\left(g_{i j}\right)$.

Let $\Omega$ be strictly star-shaped with respect to $\xi \in \Omega$. We parameterize $\Omega \backslash\{\xi\}$ by means of the mapping

$$
\mathcal{L}: \partial \Omega \times(0,1) \rightarrow \mathbb{R}^{d}:\{(x, t) \mapsto \xi+(x-\xi) t\} .
$$

Notice that the "shrunk boundary" $\mathcal{L}(\partial \Omega \times\{t\})$ is indeed contained in $\Omega$ for any $t \in(0,1)$. Using the local parametrization of the boundary by $\Gamma$ and properties of the cross-product, the Jacobian of the transformation $\mathcal{L}$ can be locally identified with

$$
J(\cdot, t)=\left|\begin{array}{cccc}
\left(\partial_{1} \Gamma^{1}\right) t & \ldots & \left(\partial_{d-1} \Gamma^{1}\right) t & \Gamma^{1} \\
\vdots & & \vdots & \vdots \\
\left(\partial_{1} \Gamma^{d}\right) t & \ldots & \left(\partial_{d-1} \Gamma^{d}\right) t & \Gamma^{d}
\end{array}\right|=\left[\partial_{1} \Gamma, \ldots, \partial_{d-1} \Gamma\right] \cdot \Gamma t^{d-1} .
$$

Hence

$$
|J(u, t)|=|g|^{1 / 2}(u) h_{\xi}(\Gamma(u)) t^{d-1}
$$

for every $t \in(0,1)$ and almost every $u \in U$. By virtue of the inverse function theorem and assumption (2), we therefore conclude that $\mathcal{L}: \partial \Omega \times(0,1) \rightarrow \Omega \backslash\{\xi\}$ is indeed a diffeomorphism.

In other words, the Euclidean domain $\Omega$ with the point $\xi$ removed can be identified with the Riemannian manifold

$$
M:=(\partial \Omega \times(0,1), G),
$$


where the metric $G$ is induced by ([6). Since the coefficients of $G$ are locally given by

$$
G_{i j}:=\left(\partial_{i} \mathfrak{L}\right) \cdot\left(\partial_{j} \mathfrak{L}\right), \quad i, j \in\{1, \ldots, d\},
$$

where $\mathfrak{L}:=\mathcal{L} \circ(\Gamma \otimes 1)$ with 1 being the identity function on the interval $(0,1)$, we plainly have

$$
\left(G_{i j}(\cdot, t)\right)=\left(\begin{array}{cccc}
g_{11} t^{2} & \ldots & g_{1 d-1} t^{2} & \Gamma \cdot\left(\partial_{1} \Gamma\right) t \\
\vdots & & \vdots & \vdots \\
g_{d-11} t^{2} & \ldots & g_{d-1 d-1} t^{2} & \Gamma \cdot\left(\partial_{d-1} \Gamma\right) t \\
\Gamma \cdot\left(\partial_{1} \Gamma\right) t & \ldots & \Gamma \cdot\left(\partial_{d-1} \Gamma\right) t & |\Gamma|^{2}
\end{array}\right) .
$$

Using the relation $|G|:=\operatorname{det}\left(G_{i j}\right)=J^{2}$ and formula (7), we see that the volume element $d \mathrm{vol}$ of the manifold $M$ is decoupled as follows:

$$
d \mathrm{vol}=h_{\xi}(x) d \sigma(x) t^{d-1} d t
$$

where $d \sigma$ and $d t$ denote the respective measures on $\partial \Omega$ and $(0,1)$. At the same time, denoting by $G^{i j}$ the coefficients of the matrix inverse to $\left(G_{i j}\right)$, we locally find that

$$
G^{d d}(u, t)=h_{\xi}^{-2}(\Gamma(u)) .
$$

If $\psi$ is any differentiable function on $(0,1)$ and 1 denotes the identity function on $\partial \Omega$, then the last result implies

$$
\left|\nabla_{G}(1 \otimes \psi)\right|_{G}=h_{\xi}^{-1}\left|\psi^{\prime}\right|,
$$

where $\nabla_{G}$ and $|\cdot|_{G}$ stand for the gradient and norm on $M$, respectively.

Using the above geometric preliminaries, the Hilbert space $L^{2}(\Omega)$ can be identified with $L^{2}(M) \equiv L^{2}(\partial \Omega \times(0,1), d \mathrm{vol})$, and the Dirichlet Laplacian in the former is unitarily equivalent to the self-adjoint operator associated in the latter with the quadratic form

$$
Q[\Psi]:=\left\|\left|\nabla_{G} \Psi\right|_{G}\right\|_{L^{2}(M)}^{2}, \quad \Psi \in D(Q):=H_{0}^{1}(M) .
$$

Recall that the Sobolev space $H_{0}^{1}(M)$ is the completion of $C_{0}^{\infty}(\partial \Omega \times(0,1))$ with respect to the norm $\left(Q[\cdot]+\|\cdot\|_{L^{2}(M)}^{2}\right)^{1 / 2}$. Let $\psi$ be a non-zero function from $H_{0}^{1}\left((0,1), t^{d-1} d t\right)$ and let 1 denote the identity function on $\partial \Omega$. Then the function $1 \otimes \psi$ belongs to the form domain $D(Q)$, and we can use it as a test function in the variational formulation for the first eigenvalue of the operator associated to $Q$. Employing (8) and (9), we get

$$
\lambda_{1}(\Omega) \leq \frac{Q[1 \otimes \psi]}{\|1 \otimes \psi\|_{L^{2}(\Omega)}^{2}}=\frac{\int_{\partial \Omega} h_{\xi}(x)^{-1} d \sigma(x)}{\int_{\partial \Omega} h_{\xi}(x) d \sigma(x)} \frac{\int_{0}^{1}\left|\psi^{\prime}(t)\right| t^{d-1} d t}{\int_{0}^{1}|\psi(t)|^{2} t^{d-1} d t}=: \lambda_{1}(\Omega ; \psi, \xi) .
$$

Now, if $\Omega$ is the ball of radius 1 centered at $\xi$, the above result reduces to

$$
\lambda_{1}\left(B_{1}\right) \leq \frac{\int_{0}^{1}\left|\psi^{\prime}(t)\right| t^{d-1} d t}{\int_{0}^{1}|\psi(t)|^{2} t^{d-1} d t} .
$$

But we know that the equality sign holds if, and only if, $\psi$ is chosen as the radial component of the first eigenfunction of the Dirichlet Laplacian in the ball. Indeed, this eigenfunction is radially symmetric and can be written as $1 \otimes \psi$ in our 
coordinates. Consequently,

$$
\min _{\psi} \lambda_{1}(\Omega ; \psi, \xi)=\lambda_{1}\left(B_{1}\right) \frac{\int_{\partial \Omega} h_{\xi}(x)^{-1} d \sigma(x)}{\int_{\partial \Omega} h_{\xi}(x) d \sigma(x)} .
$$

Minimizing the integral in the numerator with respect to $\xi$, we arrive at the quantity $F(\Omega)$ of Theorem 3, It remains to realize that the integral of support function is actually independent of $\xi$ because, by (8) and Fubini's theorem,

$$
|\Omega|=\int_{\partial \Omega \times(0,1)} d \mathrm{vol}=\frac{1}{d} \int_{\partial \Omega} h_{\xi}(x) d \sigma(x) .
$$

This concludes the proof of Theorem 3 ,

\section{Applications}

4.1. Torsional rigidity. The method of the present paper applies to other Sobolevinequality-type problems, too. For instance, let $P(\Omega)$ be the torsional rigidity of $\Omega$ defined by

$$
\frac{1}{P(\Omega)}:=\inf _{\psi \in H_{0}^{1}(\Omega) \backslash\{0\}} \frac{\|\nabla \psi\|_{L^{2}(\Omega)}^{2}}{\|\psi\|_{L^{1}(\Omega)}^{2}} .
$$

Then, following the lines of Section 3 , we have

Proposition 1. Let $\Omega$ be a bounded strictly star-shaped domain in $\mathbb{R}^{d}$ with locally Lipschitz boundary $\partial \Omega$. Then

$$
\frac{1}{P(\Omega)} \leq \frac{\left|B_{1}\right|}{P\left(B_{1}\right)} \frac{F(\Omega)}{d|\Omega|^{2}} .
$$

Again, the equality is attained for $\Omega$ being a ball. For $d=2$ this result coincides with the Pólya-Szegö bound [PS, Sec. 5.5].

4.2. The growth of the isoperimetric constant. We shall now prove Theorem 1. The idea is to estimate the inradius appearing in the bound of Theorem 2 by means of the following lower bound due to Protter $[\mathrm{Pr}]$

$$
\lambda_{1}(\Omega) \geq \frac{\pi^{2}}{4 \rho_{\Omega}^{2}} .
$$

(Protter's bound actually includes an extra term depending on the diameter, but for our purposes it is sufficient to consider the expression above.) This leads to

$$
\frac{|\partial \Omega|}{d|\Omega|} \geq \sqrt{\frac{\lambda_{1}(\Omega)}{\lambda_{1}\left(B_{1}\right)}} \frac{\pi}{2 \sqrt{\lambda_{1}\left(B_{1}\right)}},
$$

which is equivalent to the inequality of Theorem 1 due to the scaling properties $\lambda_{1}(B)=\lambda_{1}\left(B_{1}\right) r^{-2}$ and $|B|=\left|B_{1}\right| r^{d}$, where $r$ is the radius of $B$, and $\left|\partial B_{1}\right|=d\left|B_{1}\right|$.

Since Protter's bound used in the proof is not sharp for the ball, the inequality in Theorem 1 is not an improvement upon the classical isoperimetric inequality for $\lambda_{1}(\Omega) / \lambda_{1}(B)$ close to one. However, it is clear that this will be the case when this ratio becomes large. At the same time, our bound is optimal in the sense that there exist $d$-dimensional domains for which the growth of $\sqrt{\lambda_{1}(\Omega) / \lambda_{1}(B)}$ cannot 
be improved. More precisely, it is not difficult to obtain that if $\mathcal{R}$ is a $d$-dimensional parallelipiped we have

$$
\frac{|\partial \mathcal{R}|}{|\mathcal{R}|^{1-1 / d}} \leq \frac{|\partial B|}{|B|^{1-1 / d}} \sqrt{\frac{\lambda_{1}(\mathcal{R})}{\lambda_{1}(B)}} \frac{2 \sqrt{\lambda_{1}\left(B_{1}\right)}}{\pi \sqrt{d}} .
$$

The question of the optimal constant multiplying $\sqrt{\lambda_{1}(\Omega) / \lambda_{1}(B)}$ in Theorem 1 remains open.

4.3. The second eigenvalue and the gap. Combining the bound in Theorem 2 with the Ashbaugh-Benguria bound $\mathrm{AB}$ for the spectral quotient $\lambda_{2}(\Omega) / \lambda_{1}(\Omega)$ gives a similar upper bound for the second eigenvalue of a convex domain. This, together with the Faber-Krahn inequality, yields, in turn, an upper bound for the spectral gap.

Proposition 2. Let $\Omega$ be a bounded convex domain of $\mathbb{R}^{d}$. Then the second Dirichlet eigenvalue $\lambda_{2}(\Omega)$ satisfies

$$
\lambda_{2}(\Omega) \leq \lambda_{2}\left(B_{1}\right) \frac{|\partial \Omega|}{d \rho_{\Omega}|\Omega|}
$$

As a consequence, the spectral gap satisfies

$$
\lambda_{2}(\Omega)-\lambda_{1}(\Omega) \leq \lambda_{2}\left(B_{1}\right) \frac{|\partial \Omega|}{d \rho_{\Omega}|\Omega|}-\lambda_{1}\left(B_{1}\right)\left(\frac{\left|B_{1}\right|}{|\Omega|}\right)^{2 / d} .
$$

For simplicity we used the bound for convex sets, but of course the bound in Theorem 3 provides a better result valid for star-shaped domains. Note also that both upper bounds give equality for the ball. For a numerical study regarding upper and lower bounds of the gap see AF2, which also contains some new conjectures for this problem.

\section{Discussion OF THE UPPER BOUNDS AND EXAMPLES}

In spite of the fact that due to the existence of Rayleigh's variational formulation upper bounds for the first eigenvalue are, in principle, easier to obtain than lower bounds, it is slightly more delicate to obtain sharp upper bounds depending on the gometric quantities used here and which are valid in arbitrary dimensions. We shall thus now compare Theorems 2 and 3 to other existing bounds and conjectures for some particular examples.

With this in mind we begin by recalling some two-dimensional bounds. Among these there is the family of upper bounds based on the method of parallel coordinates, which includes Pólya's 1960 bound for simply connected domains $\mathrm{P}$ (sharp asymptotically on infinite rectangular strips)

$$
\lambda_{1}(\Omega) \leq \frac{\pi^{2}}{4} \frac{|\partial \Omega|^{2}}{|\Omega|^{2}},
$$

and its improvement by Payne and Weinberger [PW], namely,

$$
\lambda_{1}(\Omega) \leq \frac{\pi j_{0,1}^{2}}{|\Omega|}\left[1+\left(\frac{1}{J_{1}^{2}\left(j_{0,1}\right)}-1\right)\left(\frac{|\partial \Omega|^{2}}{4 \pi|\Omega|}-1\right)\right] .
$$

Here $J_{1}$ and $j_{0,1}$ denote, respectively, the Bessel function of the first kind of order one, and the first positive zero of $J_{0}$, the Bessel function of the first kind of order 
zero. The Payne-Weinberger bound (10) is an explicit expression in terms of the area and perimeter which is obtained from their stronger bound

$$
\lambda_{1}(\Omega) \leq \frac{4 \pi^{2}}{|\partial \Omega|^{2}} k(p)^{2}, \quad p:=1-\frac{4 \pi|\Omega|}{|\partial \Omega|^{2}},
$$

where $k=k(p)$ is the first zero of the transcendental equation

$$
J_{0}(k) Y_{1}(p k)=Y_{0}(k) J_{1}(p k) .
$$

Here $Y_{0}$ and $Y_{1}$ denote the Bessel functions of the second kind of order zero and one, respectively. It is this stronger bound that we will consider throughout this section for comparison, and we shall refer to it as the PW-bound. Note that (11) is sharp for the disc and asymptotically for infinite rectangular strips. While a generalization of Pólya's bound to arbitrary dimensions can be found in [S], the proof of the stronger result (11) does not seem to have a straightforward extension to higher dimensions.

We also remark that although (10) does give equality on the disc, the numerical study carried out in AF1 suggests that this bound might still be improved, and it is conjectured there that the optimal bound depending explicitly on the area and the perimeter and valid for simply-connected two-dimensional domains should be

$$
\lambda_{1}(\Omega) \leq \frac{\pi j_{01}^{2}}{|\Omega|}+\frac{\pi^{2}}{4} \frac{|\partial \Omega|^{2}-4 \pi|\Omega|}{|\Omega|^{2}},
$$

providing equality not only for the disc but also asymptotically on infinite rectangular strips.

Along different lines, Maz'ya and Shubin have recently proved upper and lower bounds depending on the interior capacity radius [MS].

We shall now consider some examples for which we compare the upper bounds given by Theorems 2 and 3 with the PW-bound (11) and conjecture (12).

Example 1 (Rectangular parallelepipeds). Given positive numbers $a_{1}, \ldots, a_{d}$, let $\mathcal{R}:=\left(-a_{1}, a_{1}\right) \times \cdots \times\left(-a_{d}, a_{d}\right)$. Elementary calculations show that the infimum in the definition of $F(\mathcal{R})$ is attained for the intuitive choice $\xi=0$, with the result

$$
F(\mathcal{R})=|\mathcal{R}|\left(a_{1}^{-2}+\cdots+a_{d}^{-2}\right) .
$$

For rectangles, conjecture (12) is better than the PW-bound (11) for all the values of the parameter $c:=a_{1} / a_{2} \in(0,1]$. Theorem 3 (respectively Theorem 2) provides a better upper bound than conjecture (12) in the range of $c \in(0.3,1]$ (respectively $c \in(0.7,1])$. The largest discrepancy between the upper bound of Theorem 3 (respectively conjecture (12) ) and the actual value of $\lambda_{1}(\mathcal{R})$ is in the limit $c \rightarrow 0$ (respectively for $c=1$ ) when it is about $15 \%$ (respectively $26 \%$ ).

Example 2 (Ellipsoids). Given positive numbers $a_{1}, \ldots, a_{d}$, let $\mathcal{E}$ be the domain enclosed by an ellipsoid, i.e. the surface determined by the implicit equation $f(x):=$ $\left(x_{1} / a_{1}\right)^{2}+\cdots+\left(x_{d} / a_{d}\right)^{2}-1=0$. First of all, by symmetry, it is possible to conclude that the infimum in the definition of $F(\mathcal{E})$ is attained for $\xi=0$. Since $\nabla f /|\nabla f|$ is either $+N$ or $-N$ uniformly on $\partial \Omega$, we have

$$
h_{0}^{-1}(x)=N(x) \cdot \frac{\nabla f(x)}{x \cdot \nabla f(x)}=N(x) \cdot\left(\frac{x_{1}}{a_{1}^{2}}, \ldots, \frac{x_{d}}{a_{d}^{2}}\right) .
$$

Now using the divergence theorem, we arrive at

$$
F(\mathcal{E})=|\mathcal{E}|\left(a_{1}^{-2}+\cdots+a_{d}^{-2}\right) .
$$


That is, we formally obtain the same upper bound as in the case of parallelepipeds (notice that the volume terms in the bound of Theorem 3 cancel). However, the present bound is better because $\mathcal{E} \subset \mathcal{R}$, so that $\lambda_{1}(\mathcal{E}) \geq \lambda_{1}(\mathcal{R})$ by monotonicity of Dirichlet eigenvalues.

For ellipses, Theorem 3 provides a better upper bound than conjecture (12) (which is again better than the PW-bound (11)) for all the values of the parameter $c:=a_{1} / a_{2} \in(0,1]$. Theorem 2 provides a better upper bound than conjecture (12) in the regime of $c \in(0,0.1]$. In fact, the upper bound of Theorem 3 for twodimensional ellipses seems to be familiar in the applied sciences, and it is known that for $c \geq 0.5$ the discrepancy between this and $\lambda_{1}(\mathcal{E})$ does not exceed $1 \%$ ( $c f$. Po, Sec. 7.3.4-3]).

Finally, let us mention that we obtain the same formula for $F(\mathcal{E})$ (and therefore for the upper bound of Theorem (3) also in the case when $\mathcal{E}$ is a tube of elliptical cross-section, i.e. the domain determined by $f\left(x_{1}, \ldots, x_{d-1}, 0\right)=0$ and $x_{d} \in\left(-a_{d}, a_{d}\right)$.

Example 3 (Stadium). Given positive numbers $a$ and $b$, let $\mathcal{S} \subset \mathbb{R}^{2}$ be the union of the rectangle $(-b, b) \times(-a, a)$ and two discs of radius $a$ centered at the points $(-b, 0)$ and $(b, 0)$. We put $c:=b / a \in[0,+\infty)$. Again, by symmetry, it is possible to conclude that the infimum in the definition of $F(\mathcal{S})$ is attained for $\xi=0$. Since the boundary of $\mathcal{S}$ is composed of straight and arc segments, the integral of the inverse of the support function can be computed explicitly:

$$
F(\mathcal{S})=\left\{\begin{array}{lll}
4 c+\frac{8}{\sqrt{1-c^{2}}} \arctan \sqrt{\frac{1-c}{1+c}} & \text { if } & c<1, \\
8 & \text { if } & c=1 \\
4 c+\frac{4}{\sqrt{c^{2}-1}} \log \left(c+\sqrt{c^{2}-1}\right) & \text { if } & c>1 .
\end{array}\right.
$$

In this example, Theorem 3 provides a better upper bound than conjecture (12) (which is again better than the PW-bound (11)) for $c \in[0,2.6]$, while Theorem 2 is worse than both the conjecture and PW-bound for all the values of the parameter.

It is also possible to consider the asymmetric domain $\left\{\left(x_{1}, x_{2}\right) \in \mathcal{S} \mid x_{1}>0\right\}$. Then the position of $\xi$ minimizing the infimum in the definition of $F(\mathcal{S})$ significantly depends on the value of $c$.

Example 4 (Swiss cross). As an example of a non-convex domain (but strictly starshaped with respect to the origin), let $\mathcal{C} \subset \mathbb{R}^{2}$ be the union of the two rectangles $(-b-a, b+a) \times(-a, a)$ and $(-a, a) \times(-b-a, b+a)$. We put $c:=b / a \in[0,+\infty)$. An explicit calculation yields

$$
F(\mathcal{C})=8 \frac{1+c+c^{2}}{1+c}
$$

In this example, Theorem 3 provides a better upper bound than conjecture (12) (which is again better than the PW-bound (111)) for $c \in[0,3.8]$. The case $c=2$ was numerically analysed in $[\mathrm{HS}$, and it was shown that the discrepancy between the bound and $\lambda_{1}(\mathcal{C})$ is less than $39 \%$. 


\section{REFERENCES}

[A] M. I. Aissen, A set function defined for convex plane domaines, Pacific J. Math. 8 (1958), 383-399. MR0123968 (23:A1289)

[AF1] P. Antunes and P. Freitas, New bounds for the principal Dirichlet eigenvalue of planar regions, Exp. Math. 15 (2006), 333-342. MR2264470(2007e:35039)

[AF2] P. Antunes and P. Freitas, A numerical study of the spectral gap, J. Phys. A: Math. Theor. 41 (2008), 055201.

[AM1] T. M. Apostol and M. A. Mnatsakanian, Polygons circumscribing circles, Amer. Math. Month. 111 (2004), 853-863. MR2104691 (2005g:51025)

[AM2] T. M. Apostol and M. A. Mnatsakanian, Solids circumscribing spheres, Amer. Math. Month. 113 (2006), 521-540. MR2231137 (2007c:51018)

[AB] M. Ashbaugh and R. Benguria, A sharp bound for the ratio of the first two eigenvalues of the Dirichelt Laplacian and extensions, Ann. Math. 135 (1992), 601-628. MR1166646 (93d:35105)

[BC] J. Bertrand and B. Colbois, Capacité et inéqualité de Faber-Krahn dans $\mathbb{R}^{n}$, J. Funct. Anal. 232 (2006), 1-28. MR2200165 (2007c:31005)

[BZ] Yu. D. Burago and V. A. Zalgaller, Geometric Inequalities, Springer, Berlin, 1988. MR936419 (89b:52020)

[EE] D. E. Edmunds and W. D. Evans, Spectral Theory and Differential Operators, Oxford University Press, New York, 1987. MR929030 (89b:47001)

[FMP] N. Fusco, F. Maggi and A. Pratelli, Stability estimates for certain Faber-Krahn, isocapacitary and Cheeger inequalities, to appear in Annali della Scuola Normale Superiore di Pisa, Classe di Scienze.

[G] H. Guggenheimer, Concave solutions of a Dirichlet problem, Proc. Amer. Math. Soc. 40 (1973), 501-506. MR0330481 (48:8818)

[HS] J. Hersch and W. Sawyer, Numerical implementation of coherence for the example of the "Swiss cross", Numer. Math. 59 (1991), 659-665. MR.1128461(92j:65162)

[MS] V. Maz'ya and M. Shubin, Can one see the fundamental frequency of a drum?, Lett. Math. Phys. 74 (2005), 135-151. MR.2191951 (2006m:58050)

[M] A. Melas, The stability of some eigenvalue estimates, J. Differential Geom. 36 (1992), 19-33. MR:1168980 (93d:58178)

$[\mathrm{PW}]$ L. E. Payne and H. F. Weinberger, Some isoperimetric inequalities for membrane frequencies and torsional rigidity, J. Math. Anal. Appl. 2 (1961), 210-216. MR0149735 (26:7220)

[P] G. Pólya, Two more inequalities between physical and geometrical quantities, J. Indian Math. Soc. (N.S.) 24 (1960), 413-419 (1961). MR0133059(24:A2895)

[PS] G. Pólya and G. Szegö, Isoperimetric Inequalities in Mathematical Physics, Annals of Mathematics Studies, 27, Princeton University Press, Princeton, NJ, 1951. MR0043486 (13:270d)

[Po] A. D. Polyanin, Handbook of Linear Partial Differential Equations for Engineers and Scientists, Chapman \& Hall/CRC, Boca Raton, FL, 2002. MR1935578 (2003i:35001)

[Pr] M. H. Protter, A lower bound for the fundamental frequency of a convex region, Proc. Amer. Math. Soc. 81 (1981), 65-70. MR589137(82b:35113)

[S] A. Savo, Lower bounds for the nodal length of eigenfunctions of the Laplacian, Ann. Global Anal. Geom. 19 (2001), 133-151. MR.1826398 (2002g:58055)

Department of Mathematics, Faculdade de Motricidade Humana (TU Lisbon) and Group of Mathematical Physics, University of Lisbon, Complexo Interdisciplinar, Av. Prof. Gama Pinto 2, P-1649-003 Lisboa, Portugal

E-mail address: freitas@cii.fc.ul.pt

Department of Theoretical Physics, Nuclear Physics Institute, Academy of SciENCES, 25068 ŘEž, CZech Republic

E-mail address: krejcirik@ujf.cas.cz 